Isolated sheep ventricular and atrial myocytes were labelled with a monoclonal mouse antibody against RyR. A secondary antibody of Alexa 647 allowed image acquisition using the Nikon super resolution N-STORM 4.0 microscope. We used photo-switchable fluorescent labels and acquired blinks over 10000 frames in order to resolve RyR clusters. In both the atria and ventricle, the RyRs were predominantly arranged along the z-lines with few longitudinal projections. However, we found distinct differences in the size and distance between these clusters. The RyRs in the ventricle are arranged into distinct clusters. In the atria, on the other hand, the RyR are more continuous along the z-line with less obvious cluster formation.

The use of super resolution allowed in-depth examination of the RyR in both the atria and ventricle. Our data suggests that there are chamber differences with respect to RyR distribution. This may account for the differences in calcium release mechanisms.
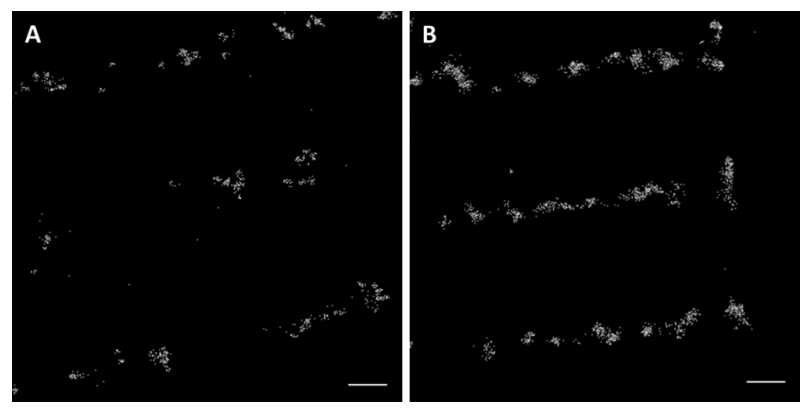

Abstract 231 Figure 1

Typical images of ryanodine receptors in sheep ventricle (A) and atria (B) obtained via super resolution STORM (stoichiometric optical reconstruction microscopy) imaging. Scale bar: $0.5 \mu \mathrm{m}$.

\section{2 \\ ENDOTHELIAL SHIP2 KNOCKOUT CAUSES NOX2 NADPH OXIDASE-DEPENDENT OXIDATIVE STRESS AND ENDOTHELIAL DYSFUNCTION}

${ }^{1}$ Peysh Patel ${ }^{*}$, ${ }^{2}$ Nicole Watt, ${ }^{2}$ Matthew Gage, ${ }^{2}$ Nadira Yuldasheva, ${ }^{2}$ Hema Viswambharan, Andrew Walker, ${ }^{2}$ Noman Ali, ${ }^{2}$ Stacey Galloway, ${ }^{2}$ Romana Mughal, ${ }^{2}$ Stephen Wheatcroft, ${ }^{2}$ Stephane Schurmans, ${ }^{2}$ Mark Kearney, ${ }^{2}$ Richard Cubbon. 'Leeds Institute of Cardiovascular and Metabolic Medicine; ${ }^{2}$ University of Liege

\subsection{6/heartjnl-2017-311726.230}

Introduction Insulin-resistant type 2 diabetes mellitus (DM) leads to premature death and disability, primarily as a consequence of accelerated vascular disease. Shc homology 2-containing inositol 5 phosphatase-2 (SHIP2) is a lipid phosphatase that suppresses insulin signalling downstream of phosphoinositide-3-kinase (PI3K). Inhibition of SHIP2 has been proposed as a therapy for type II DM, but the potential impact on vascular function is currently undefined.

Methods Mice with endothelium-specific deletion of the SHIP2 catalytic domain (ECSHIP2/+) were generated by crossing mice with LoxP sites flanking exons 18-19 of the Inppl gene with Tie2-Cre mice. Pulmonary endothelial cells (PECs) were isolated from lungs using anti-CD146 microbeads. SHIP2 knockdown was studied in human umbilical vein endothelial cells (HUVECs) by transducing with SHIP2 (or control) shRNA. Immunoblotting was performed in basal conditions and after insulin stimulation, using appropriate primary antibodies. Superoxide generation was quantified using dihydroethidium (DHE) fluorescence, and endothelial nitric oxide synthase (eNOS) activity via a 13C-l-arginine to 13C-l-citrulline conversion assay (quantified as $\%$ of baseline). Aortic hydrogen peroxide abundance was assessed using an Amplex Red assay. Vasomotor function was studied ex vivo in aortic rings. Statistical analysis was performed with unpaired and paired t-tests assuming unequal variance, with significance defined by $\mathrm{p}$-values $<0.05$.

Results PECs derived from ECSHIP2Ã $\square$ â€ $\square /+$ mice had increased basal and insulin-stimulated activation of downstream signalling intermediates, including pPDK1, S473 pAKT and S1177 peNOS. Nox2 protein expression was increased (1.19 fold; $\mathrm{p}=0.04)$, in association with increased superoxide abundance $(2.20$ fold; $p=0.004)$. This was normalised both with the Nox2 specific inhibitor Gp91ds-tat $(22 \%$ reduction; $\mathrm{p}=0.05)$ and PI3K inhibitors, Wortmannin (35\% reduction; $\mathrm{p}=0.03)$ and LY294002 $(26 \%$ reduction; $\mathrm{p}=0.03)$. SHIP2 transduction in HUVECs achieved $70 \%$ knockdown. Findings were recapitulated, with more abundant $\$ 473$ pAKT and S1177 peNOS. Moreover, there was enhanced Nox2 expression (1.39 fold; $p=0.41)$ and superoxide generation (1.42 fold; $\mathrm{p}=0.02)$, which was suppressed in the context of Nox2 (34\% reduction; $\mathrm{p}=0.03$ ) and $\mathrm{PI} 3 \mathrm{~K}$ inhibition (39\% reduction; $\mathrm{p}=0.01$ [Wortmannin], 13\% reduction; $\mathrm{p}=0.05$ [LY294002]). eNOS activity was reduced in ECSHIP2/+ PECs treated with insulin $(114 \%[ \pm 6]$ vs $136 \%[ \pm 3] ; p=0.01)$. Aortic rings from ECSHIP2/+ mice had blunted insulin-mediated vasodilation, increased hydrogen peroxide abundance, and impaired vasoconstriction in response to the non-selective NOS inhibitor L-NMMA (indicating reduced NO bioavailability).

Conclusions Endothelial-specific SHIP2 inactivation causes PI3K- and Nox2-dependent oxidative stress and endothelial dysfunction. These data suggest that SHIP2 may not be an ideal therapeutic target for diabetes-associated vascular disease.

\section{ANTI-ARRHYTHMIC ROLE OF SPHINGOSINE 1- PHOSPAHTE IN POST-OPERATIVE ATRIAL FIBRILLATION BY PAK1 ACTIVATION}

${ }^{1}$ Naseer Ahmed*, ${ }^{1}$ Daniele Linardi, ${ }^{2}$ Sajeela Ahmed, ${ }^{2}$ Riffat Mehboob, ${ }^{1}$ Giovanni Battista Luciani, ' Giuseppe Faggian, ${ }^{1}$ Alessio Rungatscher. 'University of Verona, Division of Cardiac Surgery; ${ }^{2}$ King Edward Medical University, Biomedical Sciences

\subsection{6/heartinl-2017-311726.231}

Background Atrial Fibrillation (AF) is the most common arrhythmia occurring post cardiac surgery. The incidence varies and depends on the type of surgery. Postoperative AF may cause hemodynamically unstable, that increase the risk of stroke and increase mortality. Current management for prophylaxis of postoperative AF is not satisfactory.

Objectives The purpose of this study is to investigate the antiarrhythmic role of sphingosine 1-phosphate to prevent postcardiac surgery AF.

Methods Sprague-Dawley Rats (300-350grams) Obtained from Harlan Laboratories (Udine, Italy). They were fed standard rat chow, which they had access to ad libitum. Randomly categorised into two groups $n=10$ each group. One group was treated with sphingosine 1-phospahte receptor agonist fingoli$\bmod (1 \mathrm{mg} / \mathrm{kg}$, i.v) and the control group was treated with saline. Following $15 \mathrm{~min}$ of treatment, cardioplegic arrest with the support of cardiopulmonary bypass. ECG electrodes were 